\title{
Os pais como pilares para a segurança do paciente em unidade neonatal
}

\author{
Parents as pillars for patient safety in a neonatal unit \\ Padres como pilares para la seguridad del paciente em una unidad neonatal
}

Leila Patrícia de Moura', Gisela Maria Schebella Souto de Moura", Wiliam Wegner'", Louise Viecili Hoffmeister ${ }^{\prime V}$

\begin{abstract}
RESUMO
Objetivo: conhecer a experiência dos pais como estratégia de avaliação da qualidade da assistência de enfermagem. Método: pesquisa descritiva com abordagem qualitativa, desenvolvida na Unidade Neonatal de um Hospital do sul do Brasil. A coleta de dados foi realizada através de entrevistas, utilizando a técnica do incidente crítico (TIC), com 18 pais que estavam com seus filhos internados por 20 dias ou mais e que tinham previsão e plano de alta hospitalar. Após, os dados foram submetidos à análise de conteúdo. Resultados: a análise revelou fragilidades no cuidado prestado pela equipe de enfermagem em relação à administração de medicamentos, ao uso de equipamentos, à monitorização e ao posicionamento dos bebês, aos cuidados com a pele e à higiene de mãos. Conclusão: a experiência dos pais revelou elementos que integram a avaliação da assistência em enfermagem, destacando-os como pilares para a segurança do paciente.
\end{abstract}

Descritores: Neonatologia; Enfermagem; Pais; Segurança do Paciente.

\begin{abstract}
Objective: to learn the parents' experience as a strategy for assessing the quality of nursing care. Method: in this qualitative, descriptive study at the Neonatal Unit of a hospital in southern Brazil, data were collected by critical incident (Cl) interviews of 18 parents whose children had been hospitalized for 20 days or more, and whose discharge was scheduled and planned for. The data subsequently underwent content analysis. Results: data analysis revealed weaknesses in the care provided by the nursing staff as regards administration of medication, use of equipment, monitoring and positioning of babies, skin care and hand hygiene. Conclusion: The parents' experience revealed elements that enter into evaluation of nursing care, revealing parents to be mainstays of patient safety.
\end{abstract}

Descriptors: Neonatology; Nursing; Parents; Patient Safety.

\section{RESUMEN}

Objetivo: conocer la experiencia de los padres como estrategia para evaluar la calidad de la atención de enfermería. Método: en este estudio cualitativo y descriptivo en la Unidad Neonatal de un hospital en el sur de Brasil, los datos fueron recolectados por entrevistas de incidentes críticos (IC) de 18 padres cuyos hijos habían estado hospitalizados durante 20 días o más, y cuyo alta fue programada y planificada para. Los datos posteriormente se sometieron a análisis de contenido. Resultados: el análisis de datos reveló debilidades en la atención brindada por el personal de enfermería en lo que respecta a la administración de medicamentos, uso de equipos, monitoreo y posicionamiento de bebés, cuidado de la piel e higiene de manos. Conclusión: la experiencia de los padres reveló elementos que entran en la evaluación de la atención de enfermería, revelando que los padres son pilares de la seguridad del paciente.

Descriptores: Neonatología; Enfermería; Padrea; Seguridad del Paciente.

\section{INTRODUÇÃO}

A Unidade Neonatal é considerada uma área complexa dentro dos serviços de saúde, pois os neonatos estão expostos a maiores riscos devido a particularidades como sua instabilidade fisiológica e sistemas orgânicos que ainda estão em desenvolvimento, tornando a assistência oferecida nesta unidade um motivo constante de preocupação ${ }^{1,2}$.

Os eventos adversos relacionados a assistência aos neonatos internados podem ocasionar sequelas irreversíveis ao bebê, como o agravamento clínico e até a morte, sendo os mais frequentes relacionados aos distúrbios de termorregulação, glicemia, dosagem incorreta de medicação, perda acidental de cateter intravascular, lesão cutânea e infecção ${ }^{1-3}$. Neste mesmo sentido, a segurança dos neonatos perpassa por cuidados livre de danos, que visam à manutenção e restauração da estabilidade fisiológica na adaptação extrauterina, além da necessidade de se reduzir as infecções, morbidade e mortalidade destes pacientes ${ }^{3,4}$.

'Mestre em Enfermagem. Universidade Federal do Rio Grande do Sul. E-mail: leilapm04@gmail.com. ORCID: https://orcid.org/0000-0002-6948-6104.

"Doutora em Administração. Universidade Federal do Rio Grande do Sul. E-mail: gisela.moura@ufrgs.br. ORCID: https://orcid.org/0000-0002-6215-9135.

I'Doutor em Enfermagem. Universidade Federal do Rio Grande do Sul. E-mail: wegnerwiliam@gmail.com. ORCID: https://orcid.org/0000-0002-0538-9655.

IvMestre em Enfermagem. Universidade Nova de Lisboa, Portugal. E-mail: Iv.hoffmeister@ensp.unl.pt .ORCID: https://orcid.org/0000-0002-8858-5693. 
Para proporcionar um cuidado de excelência e seguro aos bebês, as instituições de saúde têm utilizado estratégias de avaliação da qualidade, como a incorporação da percepção do paciente na projeção de melhorias dos serviços ${ }^{5}$. Pesquisas defendem que as experiências dos pacientes durante sua internação podem trazer informações importantes sobre a qualidade dos cuidados e a segurança do paciente, tornando-se uma importante estratégia de avaliação dos serviços ${ }^{5,6}$.

Esta perspectiva já vem sendo abordada no Programa Nacional de Segurança do Paciente (PNSP) instituído no Brasil, onde o segundo eixo do programa discute a importância do envolvimento do cidadão na sua segurança, pois acredita-se que os pacientes e seus familiares, quando inseridos no centro dos cuidados, podem contribuir para melhorar a segurança dos cuidados que recebem e atuar como parceiros ativos na prevenção de danos evitáveis em saúde ${ }^{7}$.

Na unidade neonatal, a inserção dos pais na rotina dos cuidados beneficia o bebê em diversos aspectos, uma vez que o envolvimento dos pais com o bebê propicia a construção de um ambiente de confiança e de liberdade para questionar a equipe de saúde sobre os cuidados ao neonato. Neste sentido, acredita-se que os pais podem ser parceiros para a segurança no cuidado com o bebê, assim como podem auxiliar na identificação e prevenção de incidentes ${ }^{8,9}$.

Destaca-se, no âmbito dos cuidados prestados em unidades neonatais, a equipe de enfermagem, por ser a equipe mais próxima do paciente, que oferece assistência ao mesmo e a sua família durante 24 horas e está envolvida com todos os aspectos que circundam a internação hospitalar. Assim, considerando que a presença dos pais na unidade neonatal é um contribuinte para a segurança do paciente, o objetivo deste estudo é conhecer a experiência dos pais como estratégia de avaliação da qualidade da assistência de enfermagem em uma Unidade Neonatal.

\section{MÉTOdo}

O local pesquisado foi a Unidade Neonatal de um hospital do interior do Estado do Rio Grande do Sul, Brasil. Esta unidade possui 25 leitos, sendo 10 de Unidade de Tratamento Intensivo Neonatal (UTIN), 10 de Unidade de Cuidados Intermediários Neonatal (UCIN) e cinco leitos de Unidade de Cuidados Intermediários Canguru (UCINco) que, no entanto, não fizeram parte da pesquisa. Os participantes do estudo foram os pais que estavam com seus filhos internados na UTIN ou UCIN, no período de maio a novembro de 2018. O critério de inclusão dos pais relacionava-se ao fato dos bebês terem 20 dias ou mais de internação, previsão e plano de alta hospitalar descrito no prontuário e previamente terem internado em leito de UTIN. A indicação dos pais que se adequavam aos critérios foi realizada pelas enfermeiras da unidade.

A coleta dos dados foi realizada com a utilização da técnica do incidente crítico (TIC), um conjunto sistematizado de procedimentos, que, ao serem aplicados, permitem o registro de comportamentos específicos ${ }^{10}$. A técnica possui três componentes que caracterizam um incidente crítico que são: a vivência de uma situação por um sujeito, que irá resultar em um comportamento e, então, em uma consequência ${ }^{10}$. Os dados foram coletados de maio a novembro de 2018, por meio de entrevistas semiestruturadas realizadas com o auxílio de um roteiro que iniciava convidando o participante a pensar no período de internação de seu filho(a). Após este primeiro momento, pedia-se que o participante lembrasse do atendimento da equipe de enfermagem, e então as seguintes questões eram elaboradas: "Qual o evento (incidente) relacionado ao atendimento de enfermagem que deseja relatar?"; "Em que local o evento ocorreu?"; "Que pessoas estavam envolvidas no evento?"; "Por que este evento Ihe gerou (in)satisfação?"; "Por que este evento foi selecionado por você para relatar? (Gerou satisfação ou insatisfação?)"; "Quando este evento ocorreu?"; "O que poderia ter sido diferente?"; "Você deseja relatar outra situação?";

Os pais foram entrevistados em uma sala reservada, na própria unidade, e a média de tempo de cada entrevista variou entre 15 e 45 minutos. As entrevistas foram realizadas pela própria pesquisadora, gravadas com dispositivo móvel, e transcritas na íntegra. Todos os participantes convidados aceitaram participar das entrevistas.

Foi empregada análise de conteúdo ${ }^{11}$, realizando-se a leitura flutuante das entrevistas para buscar experiências que contivessem os três elementos que caracterizavam um incidente crítico. Todos os incidentes encontrados foram organizados em um quadro, e posteriormente interpretados observando experiências, sentimentos, expressões que apareciam com frequência e semelhança nos incidentes críticos. Neste momento, os relatos foram agrupados por unidades de sentido e de sua classificação emergiu uma categoria analítica.

Em relação aos aspectos éticos, o estudo cumpriu as exigências da Resolução no 466/2012 ${ }^{12}$, obedecendo as diretrizes e normas regulamentadoras de pesquisa envolvendo seres humanos. Foi aprovado pelo Comitê de Ética em Pesquisa da Universidade Federal do Rio Grande do Sul, com parecer no 2595150. Os pais que aceitaram participar da pesquisa assinaram o Termo de Consentimento Livre e Esclarecido. 


\section{RESULTADOS}

O grupo de 18 participantes foi composto por 17 mães e um pai, a maioria com ensino médio completo e com idade entre 20 e 43 anos. O tempo de internação dos bebês foi, no mínimo 20 dias e o máximo de 102 dias, e a idade gestacional dos bebês variou de 27 a 33 semanas.

A partir da leitura, organização e classificação dos relatos, obteve-se 43 incidentes críticos, sendo que destes, seis incidentes descreveram experiências relacionadas às fragilidades no cuidado da equipe de enfermagem que refletem na segurança do paciente, compondo a categoria analítica "Segurança do Paciente".

As experiências retratam descuidos da equipe de enfermagem em relação à assistência prestada ao bebê que foram presenciados pelos pais, tais como: erro durante a administração de medicamentos, aquecimento inadequado após o banho, profissional relutante no monitoramento da saturação periférica de oxigênio, posicionamento inadequado no bebê ocasionando perda de dispositivo de oxigênio, negligência na troca de fraldas e omissão da higiene das mãos.

O primeiro evento a ser destacado relaciona-se com incidente que ocorreu durante a administração do medicamento:

Ele estava com um volume de medicamento infundindo $0.5 \mathrm{ml}$ por hora, e essa máquina deu um pulo, $e$ começou a infundir 50 e poucos $\mathrm{ml}$ por hora, e então eles tiveram a infusão em minutos uma infusão que era em 8 horas, né? Quando eu chamei, a pessoa viu e continuou infundindo o medicamento normal, no 0,5ml/h, mas continuou, e isso me preocupou, uma quantidade excessiva de medicamento, tem que ter todo um cuidado. E eu tive que chamar a médica, e a médica pediu para parar com o medicamento [...] então, para mim, foi falta de profissionalismo ali, sabe. Não, isso, não gostei, me fez mal ver aquilo ali. (E4).

$\mathrm{O}$ aquecimento inadequado do bebê após o banho foi outro momento observado por uma mãe. De acordo com o relato, o bebê apresentou hipertermia após o banho, e o incidente crítico foi justificado pelo mau funcionamento da incubadora, porém percebe-se também uma limitação no atendimento da equipe de enfermagem, que não considerou a observação da mãe sobre os sinais que o bebê estava apresentando.

Só teve um dia que eu cheguei de noite e recém eles tinham dado banho no nenê, aí eu olhei a incubadora: Tá quente! Aí ninguém foi lá olhar. Aí, depois eu olhei, meu nenê tá muito vermelho, aí chamei as enfermeiras: Acho que tem uma coisa errada, o nenê tá muito vermelho, acho que tá muito quente! - "Não, tá tranquilo (a enfermeira disse).". Chamei de novo, ele já estava com 37.9, quase 38 de calor, aí depois trocaram a incubadora. Só achei alguém podia passar mais seguido, às vezes passa e olha, mas ninguém bota a mão lá e olha que temperatura que tá, pra ver se realmente tá tudo certo. (E3)

Em dois momentos relatados houve falha dos profissionais em relação aos dispositivos que auxiliam na prática de oxigenioterapia para o tratamento dos bebês. No primeiro relato, observa-se que a técnica de enfermagem relutou em colocar novamente o oxímetro no bebê, deixando-o sem monitoramento de sua saturação periférica de oxigênio.

Quando ele estava sem aquele "coisinha" (saturômetro) do pezinho dele, e daí uma enfermeira (nome da técnica de enfermagem) que estava com ele, eu falei para ela que ele estava sem. Ela estava sentada mexendo no telefone, dai ela me disse: "Eu estou no meu intervalo, agora não posso.". [...] Daí ela mandou a outra guria, a outra que estava trabalhando, e daí ela foi lá [...]. Eu não gostei muito. (E9)

Neste segundo relato, a mãe encontrou o bebê desajeitado na incubadora e com o suporte de oxigenação fora da via área. Percebe-se também diferenças na frequência de observação do neonato conforme o turno de trabalho:

Em duas noites seguidas eu chego lá às 21 h, ele está quase caindo da incubadora, estava com os pezinhos lá no vidro da incubadora, todo virado de lado, sem ninho, sem nada, e o "oclinhos" (de oxigênio) em cima dos olhos, estava saturando 93\%, 94\%. Eu acho que não estava muito tempo, porque, senão, tinha baixado mais a saturação [...] imagina se eu não tivesse vindo ali, ele podia ter cansado de ficar sem o "oclinhos", podia ter ficado pior, né. Eu acho que às vezes fica tempo sem ninguém olhar, eu acho que isso é mais do turno da noite, elas ficam mais sentadas, não vão muito ver, aí às vezes eu fico bem preocupada de deixar ele, isso às vezes também me deixa um pouco nervosa. (E9)

As outras duas observações relatadas destacam fragilidade no cuidado com a pele do bebê pela equipe de enfermagem, ao negligenciar ou realizar de forma brusca a troca de fralda do mesmo:

Ontem de manhã foi bem chato assim, porque meu filho estava todo "mijadinho", aí eu disse assim: (nome da técnica), tu podia trocar o bebê? - "Mãezinha, não tem muito xixi, pode deixar assim.". Aí passou uma hora eu chamei de novo: $O$ bebê está coco e xixi, daí ela disse que não precisava trocar, e disse assim: "Essa mãezinha está insistindo bastante.". Foi o que deu pra entender, que eu estava insistindo demais em cuidar muito. Foi nessa parte que me magoou bastante. (E15) 
A técnica limpou a bundinha dele, já estava bem machucado, eu saí daqui chorando, no outro dia eu cheguei e estava com uma gaze e pomada para poder sarar, isso me atacou nos "nervos". Eu não quero mais ela cuidando do meu filho. (E12)

A lavagem das mãos foi outro aspecto evidenciado em uma das experiências, quando os pais observaram que a técnica de enfermagem estava mexendo no celular e não lavou as mãos antes de manusear o bebê:

Ela (técnica de enfermagem) sempre pedia para gente lavar as mãos para não pegar nenhuma bactéria. Mas teve momentos que ela estava no celular e daí ela já levantou, não lavou as mãos e veio mexer nele (no bebê). Daí meu marido cobrou: "Não tem que lavar as mãos? Não tem que usar luva?". - "Não, eu já fiz assepsia.". Mas acho que foi um descuido, mas ele (o pai) já ficou preocupado. (E6)

De acordo com a perspectiva dos pais, os resultados encontrados apontam para a existência de descuidos na assistência oferecida aos bebês pela equipe de enfermagem. Estes descuidos ocasionaram incidentes que, em sua maioria, causaram danos temporários aos bebês, e outros que poderiam ter causado danos permanentes se não fossem alertados pelos pais.

\section{DISCUSSÃO}

Em relação ao erro de infusão de medicação percebido pelos pais, destaca-se que os eventos adversos relacionados ao processo de medicação estão entre os incidentes mais comuns nas unidades de cuidados neonatais ${ }^{13,14}$. Em estudo realizado na Alemanha, foram observadas 281 preparações de medicamentos em UTINs, encontrando-se 38 erros, sendo os mais comuns a falta de uniformização na reconstituição/diluição dos medicamentos e a velocidade incorreta da infusão das soluções ${ }^{15}$. Em outra pesquisa, dentre os cinco tipos de erros de medicação mais comumente observados na unidade neonatal, destaca-se a velocidade errada de infusão do medicamento ${ }^{16}$.

Identificar os erros que ocorrem e suas causas são importantes ferramentas para a proposição de estratégias de melhoria. Uma alternativa para melhorar a segurança na preparação e administração de medicamentos seria a realização da checagem das etapas que envolvem este processo por dois profissionais, incluindo a conferência da taxa de infusão e do volume a ser infundido programados na bomba de infusão ${ }^{17}$. No caso ocorrido na unidade pesquisada, este tipo de estratégia poderia evitar que a medicação fosse infundida com um volume inadequado. Outras estratégias são descritas na literatura, como a utilização de bombas de infusão inteligentes, a inclusão de farmacêuticos clínicos nas equipes multidisciplinares, a avaliação dos conhecimentos sobre cálculos de medicamentos para neonatologia e o uso de conectores diferenciados para cada via de administração ${ }^{18}$.

$\mathrm{O}$ aquecimento inadequado do bebê observado e comunicado à equipe de enfermagem foi outro evento destacado. Neste, o problema ocorreu por mau funcionamento do equipamento, segundo as informações trazidas pela mãe. Como qualquer equipamento tecnológico, as incubadoras neonatais podem apresentar mau funcionamento, como também, estarem sujeitas ao manuseio inadequado dos profissionais. O mau uso das incubadoras pode provocar ocorrências prejudiciais ao bebê, como a exposição à hipertermia, podendo resultar em eventos adversos irreversíveis. Assim, para que essa tecnologia resulte em assistência segura e efetiva é necessário que o manuseio do equipamento seja realizado por profissionais capacitados e que a manutenção ocorra de maneira preventiva ${ }^{19}$.

A oxigenoterapia é essencial aos bebês com disfunção respiratória, mas possui alguns complicadores quando não realizada de forma correta, como foi observado pelos pais em relação ao uso do dispositivo de oxigênio e monitorização do mesmo. Compreende-se que a equipe de enfermagem deve estar apta para prestar uma assistência adequada ao bebê que está fazendo uso de dispositivo de oxigênio, sendo necessário conhecimentos de cuidados específicos com esse tratamento para proteger o bebê de possíveis danos ${ }^{20}$.

Os pais destacam eventos relacionados a cuidados com a pele do bebê, que é bastante frágil e propensa a lesões. Para prevenir a ocorrência de lesão de pele na região perineal do bebê, a troca das fraldas deve ser realizada sempre que houver sujidade, utilizando-se pano macio ou algodão e água para limpeza da região perianal do neonato, pois o uso de produtos químicos pode causar irritação na pele, principalmente nos prematuros extremos ${ }^{21,22}$.A equipe de enfermagem é responsável pela prevenção e tratamento das lesões de pele, e a troca de fralda deve ser realizada com periodicidade curta, com higiene delicada e produtos adequados ${ }^{22}$.

A falta e/ou inadequada higiene das mãos dos profissionais percebida pelos pais é um agravante para o cuidado seguro, pois sabemos que as Infecções Relacionadas à Assistência à Saúde (IRAS) são comuns nas Unidades Neonatais e podem ser definidas como afecções que o paciente adquire enquanto recebe tratamento. No manual publicado pela Agência Nacional de Vigilância Sanitária sobre medidas de prevenção das IRAS são apontadas as medidas a serem adotadas, dentre as quais a higiene das mãos é a mais importante ${ }^{23}$.

Todos os incidentes críticos descritos demonstram a importância dos pais estarem inseridos no processo de cuidado, pois seu envolvimento pode trazer contribuições importantes para a segurança no cuidado com o bebê, e 
auxiliar na deteç̧ão e prevenção de eventos adversos ou quase falhas que não são percebidos ou notificados pela equipe assistencial| ${ }^{5,8,24}$. Quando os pais estão inseridos nos cuidados, eles se sentem parte deste cuidado, e assim podem ser considerados pilares na segurança do paciente ${ }^{9,25}$. Portanto, o enfermeiro da Unidade Neonatal necessita desenvolver ações que auxiliem os profissionais a incentivarem os pais para este envolvimento, pois a inserção da família dentro do cuidado ainda gera muitos desafios à equipe de enfermagem.

Além destas ações, é indispensável realizar estratégias voltadas à equipe de enfermagem, como atividades educativas sobre as causas e os efeitos que os incidentes podem causar à saúde do bebê, bem como conscientizá-los sobre a importância de uma assistência de qualidade e segura ${ }^{4}$. Os incidentes identificados foram encaminhados a unidade, preservando os nomes dos participantes e dos profissionais envolvidos, para que as situações relatadas pudessem servir de subsídios na melhoria do cuidado e segurança dos pacientes. Acredita-se, desta forma, que a pesquisa tenha evitado cair na armadilha da culpabilização de profissionais e tenha contribuído na perspectiva da aprendizagem a partir do incidente.

\section{Limitações do estudo}

Destaca-se o fato da pesquisadora não residir na mesma cidade onde a pesquisa foi desenvolvida, dificultando o processo de operacionalização para a coleta dos dados. Além disto, por tratar-se de um recorte de um determinado período, não se tem conhecimento de possíveis ações implementadas pelo serviço.

\section{Contribuições para a área da Enfermagem}

A segurança do paciente não era o foco inicial da pesquisa, mas a temática emergiu nas experiências vivenciadas pelos pais durante a internação de seus filhos, trazendo aspetos importantes sobre a qualidade da assistência na unidade neonatal. Isto demonstra a necessidade de aprofundar a temática em pesquisas futuras e a técnica do incidente crítico parece um caminho profícuo para descrever objetivamente situações da prática do cuidado que estão ocorrendo sob o olhar dos pais.

\section{CONSIDERAÇÕES FINAIS}

Por meio dos relatos dos pais foi possível perceber que a Unidade Neonatal pesquisada possui fragilidades em relação à segurança do cuidado aos bebês, uma vez que foram descritos incidentes críticos durante a assistência aos neonatos. Neste sentido, considera-se que as observações dos pais são uma importante estratégia para a avaliação da qualidade da assistência da equipe de enfermagem e para a prevenção de eventos adversos, tornando-os pilares para a segurança do paciente. Assim, o olhar dos pais e sua inserção no contexto do cuidado permitem o desempenho do seu papel de parceiro ativo na prevenção de danos evitáveis, conforme preconizado no PNSP.

\section{REFERÊNCIAS}

1. Tomazoni A, Rocha PK, Ribeiro MB, Serapião LS, Souza S, Manzo BF. Perception of nursing and medical professionals on patient safety in neonatal intensive care units. Rev Gaúcha Enferm. 2017 [cited 2019 Nov 23]; 38(1):e64996. DOI: http://dx.doi.org/10.1590/1983-1447.2017.01.64996.

2. Lanzillotti LS, Andrade CLT, Mendes W, Seta MH. Neonatal adverse events and near misses reported in Brazil from 2007 to 2013. Cad. Saúde Pública [online]. 2016 [cited 2019 Nov 23]; 32(9):e00100415. DOI: http://dx.doi.org/10.1590/0102-311X00100415.

3. Rodrigues FA, Wegner W, Kantorski KJC, Pedro ENR. Patient safety in a Neonatal Unit: concerns and strategies experienced by parents. Cogitare Enferm. 2018 [cited 2019 Nov 23]; (23)2: e52166. DOI: http://dx.doi.org/10.5380/ce.v23i1.52166.

4. Góis RMO, Santos AJ, Santos LHF. The safety culture as prevention of infections related to health care in neonatology: a bibliographic review. Ciências Biológicas e de Saúde Unit. 2018 [cited 2019 Nov 24]; 4(3):27-42. Available from: https://periodicos.set.edu.br/index.php/cadernobiologicas/article/view/4610/2712.

5. Wolf JA. Consumer perspectives on patient experience. The Beryl Institute. 2018 [cited 2019 Nov 24]. Available from: https://cdn.ymaws.com/www.theberylinstitute.org/resource/resmgr/consumerstudy/2018_Consumer_Paper.pdf.

6. Doyle C, Lenoxx L, Bell D. A systematic review of evidence on the links between patient experience and clinical safety and effectiveness. BMJ Open. 2013 [cited 2019 Nov 25]; 3:e001570. DOI: http://dx.doi.org/10.1136/bmjopen-2012-001570.

7. Ministério da Saúde (Br). Reference document for the National Patient Safety Program. Ministério da Saúde; Fundação Oswaldo Cruz; Agência Nacional de Vigilância Sanitária. Brasília (DF): Ministério da Saúde; 2014 [cited 2019 Nov 25]; 40 p. Available from: https://bvsms.saude.gov.br/bvs/publicacoes/documento_referencia_programa_nacional_seguranca.pdf.

8. Sousa FCP, Montenegro LC, Goveia VR, Corrêa AR, Rocha PK, Manzo BF. Family participation in Patient Safety in Neonatal Units from the nursing perspective. Texto Contexto Enferm, 2017 [cited 2019 Nov 25]; 26(3): e1180016. DOI: http://dx.doi.org/10.1590/0104-07072017001180016.

9. Silva TO, Bezerra ALQ, Paranaguá TTB, Teixeira CC. Patient involvement in the safety of care: an integrative review. Rev. Eletr. Enf. 2016 [cited 2019 Jul 10]; 18: e1173. DOI: https://doi.org/10.5216/ree.v18.33340.

10. Flanagan JC. The critical incident technique. Arq. Bras. Psic. Apl. 1973 [cited 2019 Nov 29]; 21(2). Available from: http://bibliotecadigital.fgv.br/ojs/index.php/abpa/article/viewFile/16975/15786.

11. Bardin L. Content Analysis. São Paulo: Edições. 2016 [cited 2019 Nov 29]. Available from: 
https://madmunifacs.files.wordpress.com/2016/08/anc3a1lise-de-contec3bado-laurence-bardin.pdf.

12. Conselho Nacional de Saúde (Br). Resolução no 466 de 12 de dezembro de 2012. Diretrizes e normas regulamentadoras de pesquisas envolvendo seres humanos. Brasília (DF): CNS; 2012. [cited 2019 Nov 29]. Available from: http://conselho.saude.gov.br/resolucoes/2012/Reso466.pdf.

13. Guzzo GM, Magalhães AMM, Moura GMSS, Wegner W. Medication safety in neonatology: nursing in the perspective of the ecological restorative approach. Texto Contexto Enferm. 2018 [cited 2019 Nov 29]; 27(3): e4500016. DOI: https://doi.org/10.1590/0104-070720180004500016.

14. Hoffmeister LV, Moura GMSS, Macedo APMC. Learning from mistakes: analyzing incidents in a neonatal care unit. Rev. Latino-Am. Enfermagem. 2019 [cited 2019 Nov 30]; 27:e3121. DOI: http://dx.doi.org/10.1590/1518-8345.2795.3121.

15. Hermanspann T, Liden E, Schoberer M, Fitzner C, Orlikowsky T, Marx G, Eisert A. Evaluation to improve the quality of medication preparation and administration in pediatric and adult intensive care units. Drug Healthc Patient Saf. 2019 [cited 2019 Nov 30]; 11: 11-18. DOI: https://doi.org/10.2147/DHPS.S184479.

16. Labib JR, Labib-Youssef MR, Fatah S. High alert medications administration errors in neonatal intensive care unit: A pediatric tertiary hospital experience. Turk J Pediatr. 2018 [cited 2019 Nov 30]; 60(3): 277-285. DOI: https://doi.org/10.24953/turkjped.2018.03.007.

17. Rishoej RM, Nielsen HL, Strzelec SM, Refer JF, Beck SA, Gramstrup HM, et al. Qualitative exploration of practices to prevent medication errors in neonatal intensive care units: a focus group study. Ther Adv Drug Saf. 2018 [cited 2019 Dec 03]; 9 (7): 343353. DOI: https://doi.org/10.1177/2042098618771541.

18. Matti N, Nguyen MR, Mosel C, Grzeskowiak LE. Utilization of neonatal medication error prevention strategies: a clinical practice survey of Australian and New Zealand neonatal units. Ther Adv Drug Saf. 2018 [cited 2019 Dec 03]; 9(11): 609-617. DOI: https://doi.org/10.1177/2042098618796952.

19. Costa CC, Tonete VLP, Parada CMGL. Knowledge and practices regarding the handling of neonatal incubators among nursing professionals. Acta Paul Enferm. 2017 [cited 2019 Dec 03]; 30(2): 174-180. DOI: http://dx.doi.org/10.1590/1982-0194201700027.

20. Ali RA, Obeisat SM, Tarawneh LH. Improving nursing knowledge and care for neonates with respiratory distress in Jordan. International Nursing Review. 2019 [cited 2020 May 15]; 66(3). DOI: https://doi.org/10.1111/inr.12510.

21. Faria TF, Kamada I. Skin injuries in newborns in neonatal intensive care. Enfermería Global. 2017 [cited 2019 Dec 06]; 17(1): 211236. DOI: https://doi.org/10.6018/eglobal.17.1.273671.

22. Aredes NDA, Santos RCA, Fonseca LMM. Skin care of premature newborns: integrative review. Revista Eletrônica de Enfermagem. 2017 [cited 2019 Dec 14]; 19. DOI: https://doi.org/10.5216/ree.v19.43331.

23. Kumar S, Shankar B, Arya S, Deb M, Chellani H. Healthcare associated infections in neonatal intensive care unit and its correlation with environmental surveillance. Journal of Infection and Public Health. 2018 [cited 2020 May 15]; 11(2): 275-279. DOI: https://doi.org/10.1016/j.jiph.2017.08.005.

24. Hoffmann LM, Wegner W, Biasibetti C, Peres MA, Gerhardt LM, Breigeiron MK. Patient safety incidents identified by the caregivers of hospitalized children. Rev. Bras. Enferm. 2019 [cited 2019 Nov 30]; 72(3): 707-14. DOI: https://doi.org/10.1590/0034-71672018-0484.

25. Brodsgaard A, Pedersen JT, Larsen P, Weis J. Parents' and nurses' experiences of partnership in neonatal intensive care units: A qualitative review and meta-synthesis. J Clin Nurs. 2019 [cited 2019 Nov 30]; 28(17-18): 3117-3139. DOI: https://doi.org/10.1111/jocn.14920. 